\title{
Prevention and control measures in radiology department for COVID-19
}

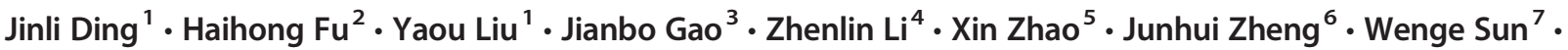 \\ Hongyan $\mathrm{Ni}^{8} \cdot$ Xinwu $\mathrm{Ma}^{9} \cdot \mathrm{Ji}^{\mathrm{Feng}}{ }^{10} \cdot{\text { Aiqin } \mathrm{Wu}^{11} \cdot \text { Jie Liu }^{3} \cdot \text { Yun Wang }}^{2} \cdot$ Pengfei Geng ${ }^{12} \cdot$ Yong Chen $^{13}$
}

Received: 5 March 2020 / Accepted: 31 March 2020 / Published online: 16 April 2020

(C) The Author(s) 2020

\begin{abstract}
Since a novel coronavirus was discovered from a cluster of patients with emerging pneumonia of unknown etiology in Wuhan, China, it has spread rapidly through droplet and contact transmission. Recently, the novel coronavirus pneumonia which was named COVID-19 by the World Health Organization (WHO) has been raised as a worldwide problem. Radiological examinations were confirmed as effective methods for the screening and diagnosis of COVID-19. It is reported that some radiologists and radiological technologists were infected when giving examinations to the patients with COVID-19. In order to reduce the infection risk of medical staff in radiology department, we summarized the experience on prevention and control measures in radiology department for COVID-19, aiming to guide the prevention and practical work for radiologists and radiological technologists.
\end{abstract}

\section{Key Points}

- The novel coronavirus spreads rapidly through droplet and contact transmission.

- Radiologists and radiological technologists were possibly infected by patients.

- Prevention and control measures in radiology department for COVID-19 are important.

Keywords Coronavirus $\cdot$ Pneumonia $\cdot$ Infection control $\cdot$ Radiology

\section{Abbreviations}

2019-nCoV Novel coronavirus 2019
COVID-19 Coronavirus disease 2019

CT Computational tomography
Haihong Fu

haihongfu@vip.sina.com

Yaou Liu

yaouliu80@163.com

$\triangle$ Jianbo Gao

cjr.gaojianbo@vip.163.com

1 Department of Radiology, Beijing Tiantan Hospital, Capital Medical University, Beijing, China

2 Department of Radiology, Peking Union Medical College Hospital, Chinese Academay of Medical Sciences, Beijing, China

3 Department of Radiology, The First Affiliated Hospital of Zhengzhou University, Zhengzhou, China

4 Department of Radiology, West China Hospital, Sichuan University, Chengdu, China

5 Department of Radiology, The Third Affiliated Hospital of Zhengzhou University, Zhengzhou, China
6 Department of Radiology, Guangdong Provincial People's Hospital, Guangzhou, China

7 Department of Radiology, The First Affiliated Hospital of China Medical University, Shenyang, China

8 Department of Radiology, Tianjin First Central Hospital, Tianjin, China

9 Equipment Department, Shandong Medical Imaging Research Institute, Jinan, China

10 Department of Radiology, Gansu Provincial Hospital, Lanzhou, China

11 Department of Radiology, The Second Affiliated Hospital and Yuying Children's Hospital of Wenzhou Medical University, Wenzhou, China

12 Department of Radiology, The Third Affiliated Hospital of Zhengzhou University, Zhengzhou, China

13 Department of Radiology, The First Hospital of Lanzhou University, Lanzhou, China 


$\begin{array}{ll}\text { DR } & \text { Digital radiography } \\ \text { HIS } & \text { Hospital information system } \\ \text { NHC } & \text { National Health Commission } \\ \text { PACS } & \text { Picture archiving and communication system } \\ \text { RIS } & \text { Radiology information system } \\ \text { WHO } & \text { World Health Organization }\end{array}$

\section{Introduction}

Since December 2019, a number of patients with pneumonia of unknown etiology have been reported in Wuhan, Hubei province [1]. It was reported that most of these patients worked at or lived around the local Huanan seafood wholesale market. Severe acute respiratory infection symptoms occurred in most patients, with some patients rapidly developing acute respiratory distress syndrome and acute respiratory failure [2]. On January 7, 2020, a novel type of coronavirus was identified from the throat swab sample of a patient, and was temporarily termed as 2019-nCoV by the World Health Organization (WHO). On February 11, the pneumonia caused by $2019-\mathrm{nCoV}$ was formally named as COVID-19 by WHO [3].

The 2019-nCoV spreads rapidly, mainly through droplet and contact transmission. Until March 3, 2020, the number of confirmed cases reached more than 80,000 according to the report from the National Health Commission (NHC) of China. COVID-19 was classified as one of the legal class B infectious diseases in China, but taking the highest level of prevention and control measures according to the "Law of the People's Republic of China on the Prevention and Treatment of Infectious Diseases."

On February 11, 2020, "Diagnosis and Treatment Protocols of COVID-19 Infection (6th edition)" was published by NHC of China [4]. It indicated that X-ray and computed tomography (CT) examinations were effective methods for the screening and diagnosis of this infectious disease [5]. However, most radiologists and radiological technologists in radiology departments had less experience when facing COVID-19 patients. Examination with nonstandard prevention procedures will lead to high risks of hospital cross-infection in the radiology department. In order to effectively reduce the infection risk of radiologists and radiological technologists, a consensus on "Prevention and control measures in radiology department for COVID-19" was presented under the endeavors of many radiologists and radiological technologists led by the Chinese Society of Imaging Technology, Chinese Medical Association, aiming to guide the prevention and practical work in the radiology department.

\section{Personal protection levels [6]}

\section{General protection}

Wearing work clothes, disposable medical masks, work caps, and gloves (if necessary).

\section{Primary level of protection}

It is suitable for pre-examination and triage, fever clinic, and infectious diseases clinic. Wearing disposable work caps, disposable medical masks (N95-type masks are recommended when contacting with confirmed patients), work clothes, isolation gowns, and disposable latex gloves (if necessary), and strictly implementing hand hygiene.

\section{Secondary level of protection}

It is suitable for medical staff who has close contact with suspected or confirmed patients. Wearing disposable work caps, protective goggles or face shields (anti-fog type), medical protective masks, protective clothing or isolation gowns, disposable latex gloves, and disposable shoe covers, and strictly implementing hand hygiene.

\section{Third level of protection}

It is suitable for aerosol-generating procedures performed for suspected or confirmed patients, such as sputum aspirating, respiratory sampling, tracheal intubation, and tracheotomy, which may cause spraying or splashing of respiratory secretions and substances of the body. Wearing disposable work caps, medical protective masks, protective face shields (fullscale respirators or positive pressure headgears are recommended), medical protective clothing, disposable latex gloves, and disposable shoe covers, and strictly implementing hand hygiene.

\section{Requirements for working environment and positions}

\section{Requirements for working environment [7]}

An independent medical imaging examination room is needed and different working areas should be strictly separated.

I. To prevent cross-infection, an independent medical imaging examination area or a dedicated radiological examination equipment (including X-ray photography equipment and CT scanner for infected patients) as well as a dedicated film printer should be available. According to the requirements for nosocomial infection prevention and control, the 
polluted area, semi-polluted area, and cleaning area need to be strictly separated and disinfected. If a dedicated examination room (such as a CT room) cannot be separated from others, strict equipment and air disinfection are required after the current patient scan and between patient encounters. Fever clinics, radiological contaminated and semi-contaminated areas, and isolation wards are the key areas for healthcare-associated infections prevention and control, which must be terminally disinfected after the examinations for confirmed patients and before the examinations for next suspected patients.

II. A dedicated radiological examinations route must be established.

III. Batch-based and time-separated services should be carried out for confirmed patients and suspected patients in fever clinic and ward, with a subsequent strict disinfection.

\section{Requirements for medical staff in radiology department [8]}

Administrator of nosocomial infections: at least one administrator needs to be designed, who is responsible for directing and supervising the disinfection and protection in the radiology department. They must guide the whole disinfection, make a clear division, and report in time to guarantee the staff and patients by avoiding to be infected by the virus.

Radiographers for bedside X-ray photography Specific radiographers need to be arranged to carry out bedside X-ray photography in key areas and take standardized photography. These radiographers are required to strictly implement the secondary level of protection. In the case of work that may cause spraying or splashing of respiratory secretions and substances of the body, such as sputum aspiration, respiratory sampling, endotracheal intubation, and tracheotomy, the third level of protection is required. After each examination, the surface of equipment should be disinfected (wiped with $75 \%$ ethanol).

Radiographers for digital radiography (DR) and CT examinations The radiographers in charge of patient position should strictly implement the secondary level of protection. The radiographers in charge of operating equipment can adopt the first or secondary level of protection.

Staff for patient registration in the key areas Patient registration should be completed by the radiographers in the key areas, and any material being in contact with the confirmed patients must be separately and safely stored. Staff for patient registration in the non-key area should take the primary level of prevention. It is recommended to make full use of the hospital information system (HIS), picture archiving and communication system (PACS), and radiology information system (RIS) to achieve paperless management.

Other staff working in the non-key areas in the radiology department It is recommended to take the general protection when working in the cleaning areas, take the primary protection when working in the semi-contaminated areas, and take the secondary protection when working in the contaminated areas. In the case of work that may cause spraying or splashing of respiratory secretions and substances of the body, such as sputum aspiration, respiratory sampling, endotracheal intubation, and tracheotomy, the third level of protection is required.

\section{Working mode for radiographers in key areas (trial)}

The radiographers for bedside X-ray photography, DR, and CT examinations working in the isolation area are recommended to take a $2+2$ working mode, due to the possibility of closely contacting with the confirmed patients. In the $2+2$ working mode, the radiographers should complete a first period of 14 workdays in the isolation area, and subsequently take a second period of 14 days in a specific dedicated isolation ward for supervised medical observation before returning to normal work.

\section{Processes of wearing and removing protective clothing $[9,10]$}

\section{Process of wearing protective clothing}

Seven steps of hand-washing; wearing a cap; wearing a medical protective mask (taking the leak test); wearing medical protective clothing (after removing shoes); wearing latex gloves (inner layer); wearing isolation clothing; wearing latex gloves (outer layer); wearing rubber boots/shoes; wearing boot/shoe covers; wearing goggles/face shield; checking tightness.

\section{Process of removing protective clothing}

In contaminated areas: clearing visible dirt; hand hygiene; taking off outer boot/shoe covers; hand hygiene; taking off isolation clothing and outer gloves; hand hygiene. In the semi-contaminated area: taking off goggles/mask; hand hygiene; taking off protective clothing, inner gloves, and boot covers; hand hygiene; taking off medical protective masks; taking off the cap; seven steps of hand-washing. 


\section{Cleaning and disinfection of radiology equipment [11]}

\section{Daily cleaning}

I. Metal surface and painted surface can be wiped with soft detergent, and then dried with dry towel. Corrosive cleaning agent, corrosive detergent, and corrosive polishing agent are not allowed.

II. Chrome parts should only be wiped with a dry towel. Abrasive polishes are not allowed. Wax is suggested to protect the surface coating. Plastic surfaces can only be cleaned with soap and water.

III. Common glass cleaner except for amide products can be used to clean the touch screen. Spray the glass cleaner on the cloth or towel, and then wipe the touch screen. Be sure to remove the liquid drops in time to prevent it from flowing into the gap of the equipment.

\section{Equipment disinfection}

I. After each examination (including X-ray photography, CT, magnetic resonance imaging, or others), the equipment must be disinfected by wiping the surface using $75 \%$ ethanol.

II. Corrosive disinfectants are not allowed.

III. Disinfectant sprays should be used carefully, as they may penetrate into the equipment, resulting in short-circuit, metal corrosion, or other damage. If the spray disinfector is needed in the room, the equipment must be shut down, cooled, and completely covered with plastic film before starting the spray disinfection. Then the equipment itself is disinfected by wiping the surface using $75 \%$ ethanol.

\section{Floor disinfection}

Visible pollutants should firstly be removed using disposable water absorption material, and then the floor should be disinfected by using $2000 \mathrm{mg} / \mathrm{L}$ disinfectant containing chlorine (except for chlorhexidine). Furthermore, any other objects (such as handrail, door handle, window, and wall switch) should also be disinfected.

\section{Air disinfection}

Air disinfection for the equipment room is recommended by using ultraviolet irradiation (continuous irradiation for more than $30 \mathrm{~min}$ ), followed by ventilation for more than $30 \mathrm{~min}$.

\section{Other disinfection}

All disposable protective products must be non-reusable. The reusable protective products (such as goggles) should be placed at the designated disinfection place, and be soaked in $1000 \mathrm{mg} / \mathrm{L}$ disinfectant containing chlorine (except for chlorhexidine) or $75 \%$ ethanol for more than $1 \mathrm{~h}$.

\section{Treatment measures of medical waste in radiology department [12]}

\section{Collection process of medical waste}

All waste from confirmed patients are regarded as infectious medical waste, and should be managed strictly according to the following process.

The process of disposing infectious waste is as follows: put the infectious waste into the medical waste collection bag (ideally be no more than $3 / 4$ full); spray the bag with $5000 \mathrm{mg} / \mathrm{L}$ disinfectant containing chlorine (except for chlorhexidine); seal the inner layer and outer layer in a GooseNeck-Type and spray the layers with $5000 \mathrm{mg} / \mathrm{L}$ disinfectant containing chlorine (except for chlorhexidine); paste special identifications in the outer layer, and store it in the specialized site for medical wastes.

\section{Personnel protection}

The cleaners should take the secondary level of protection and be responsible for handover registration, safe transportation, and proper storage of the infectious medical waste.

\section{Summary}

Radiological examination, especially chest CT, plays an irreplaceable role in the diagnosis of patients with COVID-19. Radiologists, radiological technologists, and nurses must have a great command of the individual protection and disinfection procedures when working in radiology department, especially in key areas.

Acknowledgements We thank the following experts from different hospitals in China for their supports to this paper:

Baozeng Wang, Department of Infections, Beijing Tiantan Hospital, Capital Medical University

Bing Zhang, Department of Radiology, The Affiliated Drum Tower Hospital, Medical School of Nanjing University

Chenhong Fan, Department of Radiology, Zhongnan Hospital of Wuhan University

Daoyong Liu, Department of Radiology, Beijing Children's Hospital, Capital Medical University

Dong Li, Department of Radiology, Tianjin Medical University General Hospital 
Dong Xu, Department of Radiology, Beijing Chest Hospital, Capital Medical University

Fajin Lv, Department of Radiology, The First Affiliated Hospital of Chongqing Medical University

Fei Shan, Department of Radiology, Shanghai Public Health Clinical Center

Guangping Zheng, Department of Radiology, The Third People's Hospital of Shenzhen

Haikun Du, Department of Radiology, Xining First People's Hospital

Haikuo Wang, Department of Radiology, Beijing Tiantan Hospital,

Capital Medical University

Haoliang Li, Department of Radiology, Pulmonary Hospital Affiliated to Tongji University

Hongjun Li, Department of Radiology, Beijing Youan Hospital, Capital Medical University

Housheng Ma, Department of Radiology, Yantai Yu Huang Ding Hospital

Huadan Xue, Department of Radiology, Peking Union Medical

College Hospital

Jiaojiao Liu, Department of Radiology, Beijing Youan Hospital,

Capital Medical University

Jiawen Luo, Department of Radiology, The Second Affiliated Hospital of Dalian Medical University

Jin Lv, Department of Radiology, Wuhan Jinyintan Hospital

Junhui Zheng, Department of Radiology, Guangdong Provincial

People's Hospital

Lei Shi, Department of Radiology, Shanghai Public Health Clinical Center

Li Fan, Department of Radiology, Changzheng Hospital, Second Military Medical University

Longjiang Zhang, Department of Medical Imaging, Jinling Hospital,

Medical School of Nanjing University

Min Jiang, Department of Radiology, Chengdu Public Health Clinical

Medical Center

Ming Yang, Department of Radiology, Shijiazhuang Fifth Hospital

Minjie Zhang, Department of Radiology, Children's Hospital of Fudan University

Pan Liang, Department of Radiology, The First Affiliated Hospital of Zhengzhou University

Pengfei Geng, Department of Radiology, The Third Affiliated

Hospital of Zhengzhou University

Puxuan Lu, Department of Radiology, Shenzhen Chronic Disease Prevention Center

Rengyin Zhang, Department of Radiology, Shanghai Public Health

Clinical Center

Rongmeng Jiang, Department of Infections, Beijing Ditan Hospital,

Capital Medical University

Shengyuan Lai, Department of Radiology, The Second Affiliated

Hospital of Dalian Medical University;

Shouyong Shi, Department of Radiology, Beijing Chest Hospital, Capital Medical University

Shuang Xia, Department of Radiology, Tianjin First Central Hospital Shucong Xu, Department of Radiology, Children's Hospital of Capital Institute of Pediatrics

Shuo Li, Department of Radiology, Beijing Ditan Hospital, Capital

Medical University

Songfeng Jiang, Department of Radiology, Guangzhou Eighth

People's Hospital

Wenluan Fan, Department of Radiology, Wuhan Jinyintan Hospital

Xiaopeng Du, Department of Radiology, Beijing Ditan Hospital, Capital Medical University

Xiaoyi Duan, Department of Radiology, The First Affiliated Hospital of Xi'an Jiaotong University

Xin Dai, Department of Radiology, Chongqing Public Health Medical Treatment Center
Xingui Peng, Department of Radiology, Zhongda Hospital, Medical School, Southeast University

Xiongying Yu, Department of Radiology, The Third Hospital of South

Medicine, Guangzhou City, Guangdong Province

Yanqing Fan, Department of Radiology, Wuhan Jinyintan Hospital

Yantao Niu, Department of Radiology, Beijing Tongren Hospital, Capital Medical University

Yaqin Zhang, Department of Radiology, The fifth Affiliated Hospital of Sun Yat-sen University

Yijun Zhang, Department of Radiology, Shanghai Public Health Clinical Center

Yong Zhang, Department of Radiology, Beijing Tiantan Hospital, Capital Medical University

Yuxin Shi, Department of Radiology, Shanghai Public Health Clinical Center

Zaiyi Liu, Department of Medical Imaging, Jinling Hospital, Medical School of Nanjing University

Zhenying Xia, Department of Radiology, Beijing Youan Hospital, Capital Medical University

Zhiying Yang, Medical Imaging Center, Dazhou Central Hospital

Ziqiao Lei, Department of Radiology, Union Hospital, Tongji Medical College, Huazhong University of Science and Technology

Funding information The authors state that this work has not received any funding.

\section{Compliance with ethical standards}

Guarantor The scientific guarantor of this publication is Prof. Haihong Fu.

Conflict of interest This paper obtained the "certificate of authorization" for reprint of the content from "Expert consensus of radiological examination scheme and infection prevention of the 2019 novel coronavirus pneumonia (First version)" which was in Chinese and had been accepted by a Chinese journal "Electronic Journal of Emerging Infectious Diseases."

The authors of this manuscript declare no relationships with any other companies, whose products or services may be related to the subject matter of the article.

Statistics and biometry No complex statistical methods were necessary for this paper.

Informed consent Not applicable.

Ethical approval Not applicable.

Methodology

- Multicenter study

Open Access This article is licensed under a Creative Commons Attribution 4.0 International License, which permits use, sharing, adaptation, distribution and reproduction in any medium or format, as long as you give appropriate credit to the original author(s) and the source, provide a link to the Creative Commons licence, and indicate if changes were made. The images or other third party material in this article are included in the article's Creative Commons licence, unless indicated otherwise in a credit line to the material. If material is not included in the article's Creative Commons licence and your intended use is not permitted by statutory regulation or exceeds the permitted use, you will need to obtain permission directly from the copyright holder. To view a copy of this licence, visit http://creativecommons.org/licenses/by/4.0/. 


\section{References}

1. Huang C, Wang Y, Li X et al (2020) Clinical features of patients infected with 2019 novel coronavirus in Wuhan, China. Lancet. https://doi.org/10.1016/S0140-6736(20)30183-5

2. Hui DS, Azhar EI, Madani T et al (2020) The continuing 2019nCoV epidemic threat of novel coronaviruses to global health-the latest 2019 novel coronavirus outbreak in Wuhan, China. Int J Infect Dis 91:264-246

3. World Health Organization (2020) WHO Director-General's remarks at the media briefing on 2019-nCoV on 11 February 2020. World Health Organization. Available via https://www.who.int/dg/ speeches/detail/who-director-generals-remarks-at-the-mediabriefing-on-2019-ncov-on-11-february-2020. Accessed 11 Feb 2020

4. The Novel Coronavirus Pneumonia Emergency Response Epidemiology Team (2020) The epidemiological characteristics of an outbreak of 2019 novel coronavirus diseases (Covid-19) in China. Zhonghua Liu Xing Bing Xue Za Zhi 41(2):145-151

5. Guan W, Ni Z, Hu Y et al (2020) Clinical characteristics of coronavirus disease 2019 in China. N Engl J Med. https://doi.org/10.1056/ NEJMoa2002032

6. National Health Commission of the People's Republic of China (2020) Diagnosis and Treatment Protocols of Covid-19 Infection (6th edition). General Office of the National Health Commission, Medical Letter. Accessed 18 Feb 2020
7. National Health Commission of the People's Republic of China (2020) The guidance of infection prevention and control technology for the novel coronavirus in medical institutions (1st edition). General Office of the National Health Commission, Medical Letter. Accessed 22 Jan 2020

8. National Health Commission of the People's Republic of China (2019) WS/T 313-2019 Specification of hand hygiene for healthcare workers. Standards Press of China, Beijing Accessed 26 Nov 2019

9. Ministry of Health of the People's Republic of China (2009) WS/T 311-2009 Technique standard for isolation in hospital. Standards Press of China, Beijing Issued 1st Apr 2009

10. National Health Commission of the People's Republic of China (2019) Basic system of infection prevention and control in medical institutions (trial). General Office of the National Health Commission, Medical Letter. Issued 18 May 2019

11. National Health and Family Planning Commission of the People's Republic of China (2016) WS/T512-2016 Regulation for cleaning and disinfection management of environmental surface in healthcare. Beijing: Standards Press of China. Issued 27 Dec 2016

12. Ministry of Health of the People's Republic of China (2012) WS/T 367-2012 Regulation of disinfection technique in healthcare settings. Beijing: Standards Press of China. Issued 5 Apr 2012

Publisher's note Springer Nature remains neutral with regard to jurisdictional claims in published maps and institutional affiliations. 